\title{
Electrochemical Sensing on a Nanostructured Silicon Mass Spectrometry Surface
}

\section{Tsao C-W* and Guo Z-M}

Department of Mechanical Engineering, National Central University, Taoyuan City, 32001, Taiwan

*Corresponding author: Chia-Wen Tsao, Department of Mechanical Engineering, National Central University, Taoyuan City, 32001, Taiwan

Received: April 09, 2021; Accepted: J uly 10, 2021; Published: July 17, 2021

\begin{abstract}
Mass Spectrometry (MS) is a widely used analytical tool that provides quantitive information (molecule weight and intensity) of the analyte. Nanostructured silicon-based surface-assisted desorption/ionization mass spectrometry (LDI-MS) provides matrix-free and high sensitivity advantages. However, the mass spectrometer is a large and expensive tool limiting the onsite screening or point-of-care testing applications. Electrochemical sensing, on the other hand, is a simple and less-expensive detection method that can be used as portable onsite screening purposes. If the nanostructure silicon (nSi) surface can be used for electrochemical sensing, it opens the possibility of using nSi surface for both electrochemical sensing and Mass Spectrometry (MS) detection. Therefore, in this paper, we demonstrate the feasibility of using nSi surface for electrochemical sensing. Effects of the major nSi surface process parameters, including metal-assisted etching time and electroless Au decoration/deposition time to the electrochemical was evaluated.
\end{abstract}

Keywords: Mass Spectrometry; LDI-MS; Nanostructure silicon

\section{Introduction}

Mass Spectrometry (MS) measures the mass-to-charge $(\mathrm{m} / \mathrm{z})$ ratio to identify a molecule's weight and structure. MS has been used in various fields, including analytical chemistry, forensic toxicology, proteomics, drug discovery, earth science, etc. [1-3]. Soft ionization MS methods such as Electrospray Ionization Mass Spectrometry (ESI-MS) or Matrix-Assisted Laser Desorption/Ionization Mass Spectrometry (MALDI-MS) have been commonly applied in biomolecules analysis. In ESI-MS, the analyte was ionized by applying a high potential difference (typically around $3 \sim 4 \mathrm{kV}$ ) between the capillary top and mass spectrometer orifice at atmospheric pressure. For MALDI-MS, although the molecule desorption/ionization principle is still not clearly understood, it is believed that the sample is ionized through the plus laser beam irradiation on the matrixmixed samples $[4,5]$. The off-line MALDI-MS nature also provides benefits of preserving samples on the target substrate and capable of incorporating with the microfluidics technologies through MS interfacing [6,7]. Nevertheless, MALDI-MS still suffers from matrix noise background issues that interfere in the low $\mathrm{m} / \mathrm{z}$ region. One innovative silicon-based nanotechnology called Desorption/ Ionization on Silicon Mass Spectrometry (DIOS-MS) was proposed to address this limitation [8]. In DIOS-MS, the high surface-area-ratio silicon nanostructures replace the role of matrix in MALDI absorbing the laser energy ablated from the mass spectrometer. In addition to the matrix-free advantage, DIOS-MS is also a highly sensitive method. With proper surface medication, detection sensitivity as high as attomole level can be achieved [9]. Similar to porous silicon, various nanostructured-based laser desorption/ionization mass spectrometry (LDI-MS) techniques have demonstrated. Methods such as silicon nanowires [10,11], nanostructured silicon (nSi) [12-14], germanium nanodots [15], carbon nanodots [16], silicon nanocones [17], silicon nanowell [18], silicon nanopost [19], inorganic materials [20], and nanoparticle materials [21] that include metal, carbon, or siliconbased nanomaterials and nanocomposites were reported. These nanostructured-based LDI-MS methods provide unique advantages in high sensitivity, high salt or contamination tolerance, simple for sample preparation, and rapid analysis for a wide range of applications including peptide/protein analysis [22,23], phosphopeptide [24,25], tumor imaging [26], abused drug [27], bacterial [28], and food [29] analysis.

In a recent demonstration [30], a mesoporous germanium LDIMS substrate was demonstrated to detect illicit drugs in the workplace or roadside from water or saliva samples. This method shows the potential of using nanostructured-based LDI-MS in onsite or pointof-care testing applications. Nevertheless, the mass spectrometer is a large, expensive, delicate high-end facility that fundamentally constrains its application in onsite or point-of-care testing detection. Therefore, a low-cost and straightforward onsite detection method needs to be developed to compensate for the LDI-MS limitation in onsite screening. Electrochemical biosensors provide simple, rapid, and low-cost advantages, which are ideal for quick onsite sample screening purposes [31]. The facility required for electrochemical detection is also inexpensive and portable for onsite purposes. Thus, in this paper, we demonstrate the idea of using the nanostructured silicon (nSi) platform for electrochemical sensing that also capable of performing matrix-free LDI-MS on a silicon substrate. The electrochemical sensing can be used for portable and low-cost onsite pre-screening. We focus on studying the effects of $\mathrm{nSi}$ process parameters effects to the electrochemical sensing on nSi surface.

\section{Materials and Methods}

\section{Electrochemical sensing}

Cyclic Voltammetry (CV) was used as the electrochemical sensing for $\mathrm{nSi}$. The CV curves were obtained from the Galvanostat (Autolab,
Austin J Nanomed Nanotechnol - Volume 9 Issue 1 - 2021 ISSN : 2381-8956 | www.austinpublishinggroup.com Tsao et al. (C) All rights are reserved 
PGSTAT101) where the nSi substrate was used as the working electrode, $\mathrm{Ag} / \mathrm{AgCl}$ electrode was used as the reference electrode, and platinum wire was used as counter electrode for electrochemical sensing. The glucose sample for electrochemical sensing was prepared by mixing the glucose sample with $10-3 \mathrm{M}$ potassium hexacyanoferrate (III) and potassium hexacyampferrate (II). The $1 \times 1 \mathrm{~cm}^{2} \mathrm{nSi}$ electrode was put in the glucose sample and staying for 10 minutes allowing sufficient sample reaction. The galvanostat scanning rate was set to $0.1 \mathrm{~V} / \mathrm{s}$, and step potential was set to $0.00244 \mathrm{~V}$.

\section{Nanostructured silicon surface preparation}

The nSi surface was fabricated by metal-assisted etching and two steps of electroless plating processes. The silicon substrate, P-type (100), was first cut into $1 \mathrm{~cm} \times 2 \mathrm{~cm}$ size and covered $1 \mathrm{~cm} \times 1 \mathrm{~cm}$ with SEM tape to protect this area during etching. Prior to etching, the silicon surface was ultrasonic bath clean with acetone $(99.9 \%$, electronic grade) and isopropanol alcohol (99.5\%, electronic grade) for 3 minutes, followed by a deionized water rinse and nitrogen gun blow dry. The silicon wafer was immersed in a hydrogen fluoride ( $49 \%$, electronic grade) and hydrogen tetrachloroaurate (III) trihydrate $(0.01 \mathrm{M} / 2.4 \mathrm{M})$ deposition solution for 30 seconds to deposit a thin gold film on the silicon surface. The gold-coated Si wafer was then rinsed with methanol (99.9\%, electronic grade). After gold coating, we use a metal-assisted etching process creating silicon nanostructures [32]. The silicon substrate was immersed in a hydrogen fluoride (49\% electronic grade), hydrogen peroxide (31\%, electronic grade), and ethanol (99.5\%, electronic grade) 1:1:1 volume ratio mixture for 300 900 seconds to create the nanostructures on the silicon surface, and fully rinsed with methanol (99.9\%, electronic grade) for two minutes. After silicon nanostructure fabrication, the nSi surface was decorated with gold nanoparticles by another electroless deposit step. Due to the high surface hydrophobicity $\left(>130^{\circ}\right)$ of the nSi surface, the deposition solution can't penetrate the nanostructure pores for efficient gold decoration/deposition. We modify the nSi surface into hydrophilic $\left(<20^{\circ}\right)$ by oxygen plasma treatment $\left(18 \mathrm{~W}, 7.5 \times 10^{-1}\right.$ torr,
60 seconds) prior to the deposition. The nSi surface was immersed in hydrogen tetrachloroaurate (III) trihydrate $(0.01 \mathrm{M} / 2.4 \mathrm{M})$ deposition solution for 15 seconds to decorate the gold nanoparticles on the $\mathrm{nSi}$ surface.

\section{Results and Discussion}

\section{Electrochemical Sensing on a nSi-MS surface}

The matrix-free nanostructured-based LDI-MS methods have proofed as matrix-free MS analysis platform for small molecule analysis. High detection sensitivity has been demonstrated [9] using model peptides. Compared to ESI or MALDI, nSi is a relatively simple approach. However, despite these advantages, nSi-MS still required an expensive mass spectrometer, which is not ideal for onsite or portable POCT usage. Electrochemical biosensors provide simple, rapid, and low-cost advantages, which are suitable for rapid, high throughput onsite sample screening purpose. To integrate these two techniques' capabilities, we demonstrate the idea using $\mathrm{nSi}$ surface for electrochemical sensing. In this paper, we choose glucose as a standard sample to proof of the concept. For MS detection, the samples on $\mathrm{nSi}$ surface first required to be ionized into charged molecules. For glucose samples on the nSi surface, due to the catalyst reaction. The gold nanoparticle decorated on the porous silicon surface was used to catalyze glucose into a negative charged gluconic acid. This negative charged gluconic acid enables the MS detection in to identify the glucose level negative-ion mode [14]. This matrixfree MS analysis is fully compatible with the electrochemical sensing using the same nSi surface. For electrochemical sensing, the gold nanoparticles decorated on the nanostructured silicon surface serves as a stable enzyme-free working electrode for glucose sensing through electrochemical oxidation mechanism [33]. The high surface-to-area ratio $\mathrm{nSi}$ surfaces can also effectively increase the working surface area to further enhance the electrochemical sensing sensitivity level. In the following sections, critical process parameters including etching and deposition time to surface nanostructured silicon surface
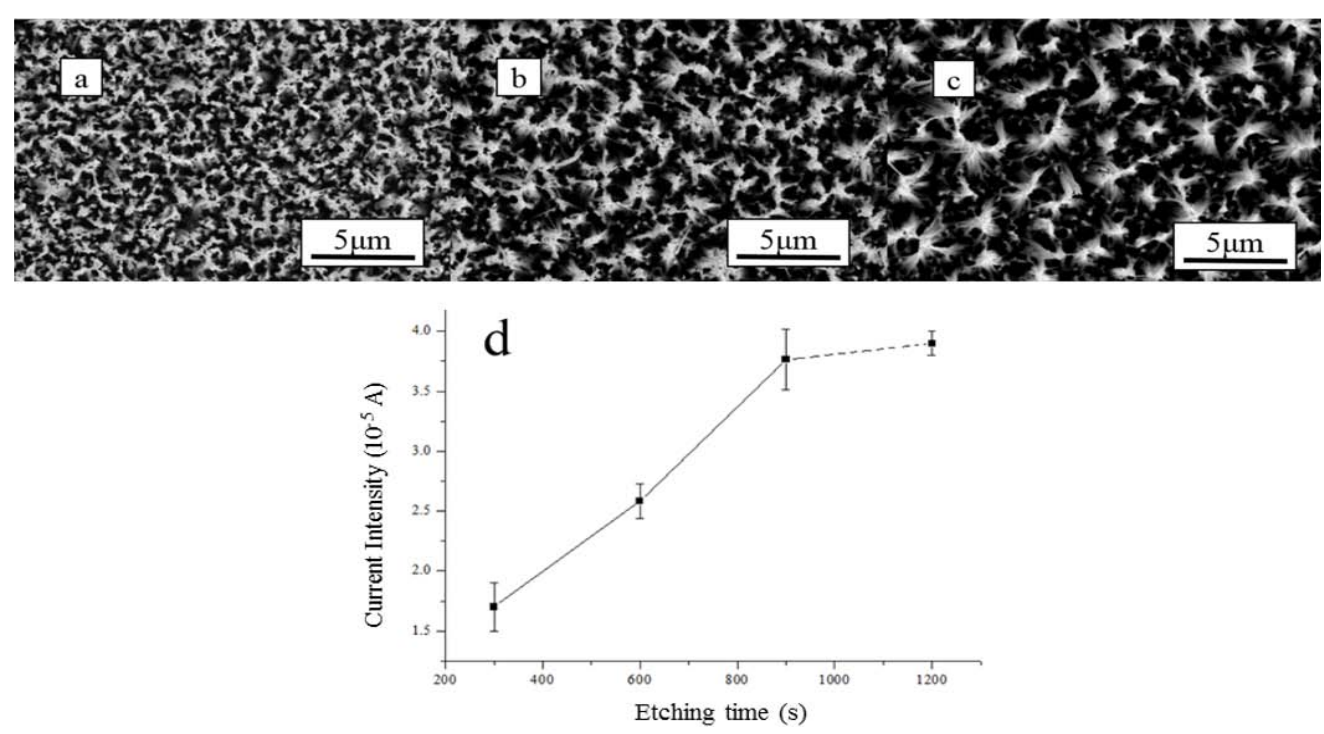

Figure 1: Top SEM images showing nSi surface morphologies with metal-assisted etching times of (a) 300 (b) 600 and (c) 900 seconds. (d) Shows electrochemical current intensity with etching time. It is noted that the first and second Au electroless deposition time were 30 and 15 seconds. The glucose sample concentration is $10^{-5} \mathrm{M}$. 

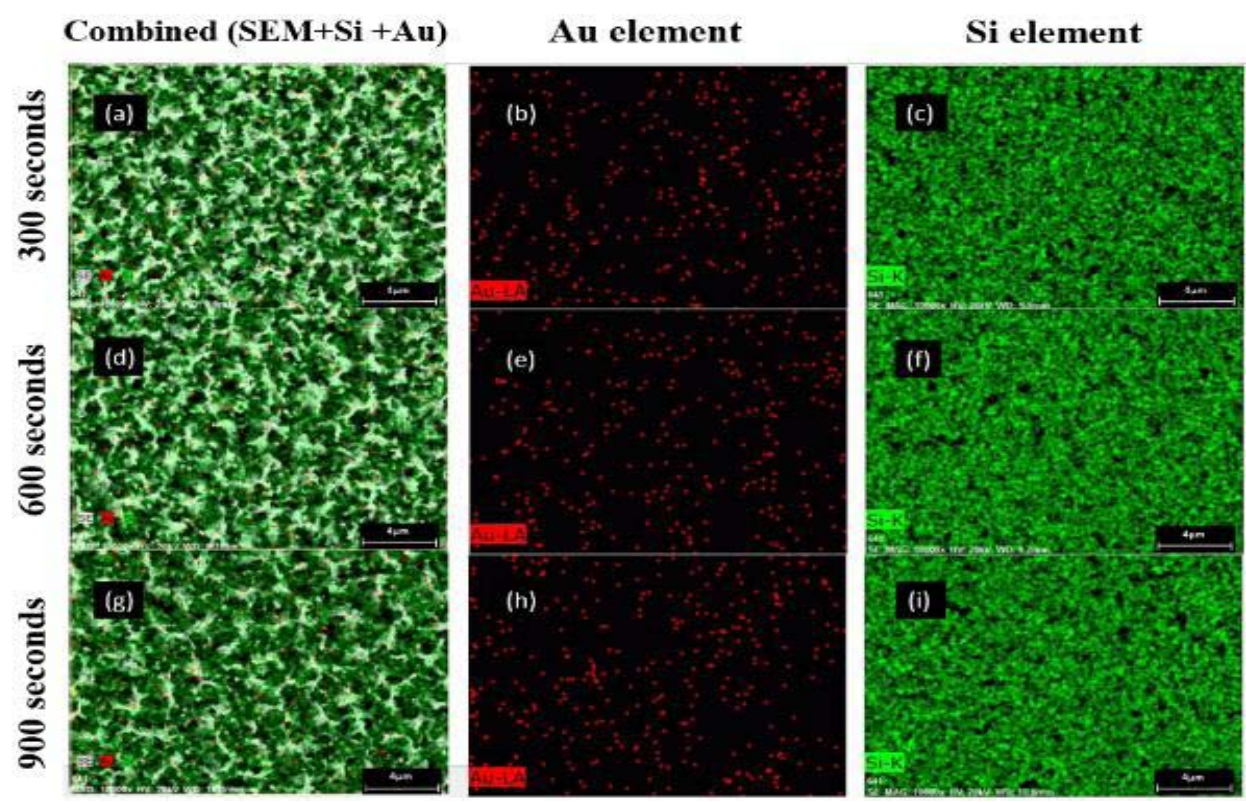

Figure 2: SEM/EDS elemental mapping of nanostructure silicon top surface with 300 seconds (a c), 600 seconds (d f), and 900 seconds ( $\mathrm{g}$ i) metal-assisted etching time.
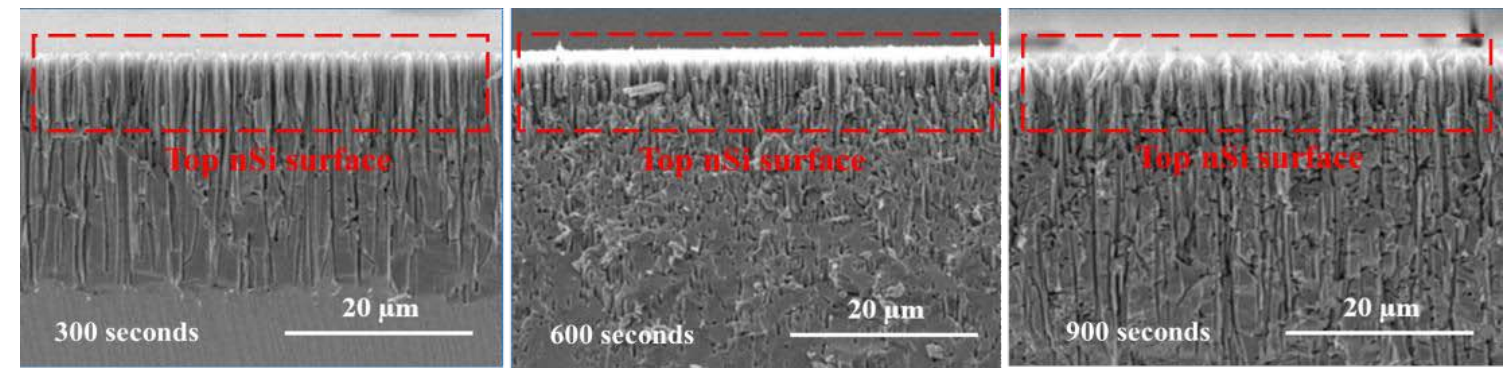

Figure 3: Cross-sectional SEM images nSi surface with different etching time.

morphology and electrochemical sensing are evaluated.

\section{Effects of metal-assisted etching time and nanostructured silicon surface morphology to electrochemical sensing}

In this section, we focus on investigating the effects of nanostructured surface morphology to the electrochemical sensing. The nSi surface was fabricated by one metal-assisted etching and two electroless $\mathrm{Au}$ deposition steps. The first $\mathrm{Au}$ electroless deposition step was used to coat a thin Au layer on the bare silicon surface for the consequent metal-assisted etching. The second Au electroless deposition was used to decorate the Au nanoparticles on the silicon nanostructures. In our experiments, for the first $\mathrm{Au}$ electroless deposition, we found excessive electroless $\mathrm{Au}$ deposition time may result in gold grows into thin films rather than gold particles. Therefore, an optimized 30 seconds was selected for the first $\mathrm{Au}$ electroless deposition steps. We also found metal-assisted etching time has significant impacts on the nSi surface morphology as well as the electrochemical sensing performance. Therefore, the metal-assisted etching time was first evaluated in this section. Figure 1a-1c shows the silicon surface with various etching time from 300 to 900 seconds. As shown in the figure, the silicon nanostructure pore size increases with the etching time from $\sim 50 \mathrm{~nm}$ (Figure 1a) to $\sim 2.0 \mu \mathrm{m}$ (Figure 1c).
The current intensity also increased from $\sim 1.7 \times 10^{-5} \mathrm{~A}$ to $\sim 3.7 \times 10^{-5}$ A for etching time from 300 to 900 seconds. According to RandlesSevcik equation, the peak current intensity potentially increased with the working electrode area. Thus, in nSi electrochemical sensing, we postulated that larger pore size promotes easy sample penetration down to the nanostructured silicon surface, which allows more $\mathrm{Au}$ nanoparticles to decorate onto the nanostructured silicon surface and catalyst reaction for enhanced current density and sensitivity level. We also test the time to 1,200 seconds. As shown in Fig 1d dash line, the current intensity only slightly increased $2.7 \%$ to $\sim 3.8 \times 10^{-5} \mathrm{~A}$, but it increased the processing time. Therefore, we choose 900 seconds etching time as optimized metal-assisted etching.

To further prove the hypothesis of larger pore sizes (increased with etching time) promotes better Au nanoparticle decoration on the nanostructure surface for electrochemical sensing. We performed the SEM/EDS elemental analysis of the nanostructured silicon surface. As shown in Figure 2, the red color presents Au element (Figure 2b, 2e \& 2h) and green color shows Si element (Figure 2c, 2f \& $2 \mathrm{i})$. The Au element increased with the pore size (etching time) from $0.47 \%$ (300 seconds), $0.74 \%$ (600 seconds) to $1.88 \%$ (900 seconds). This suggests that under identical second Au decoration/deposition 

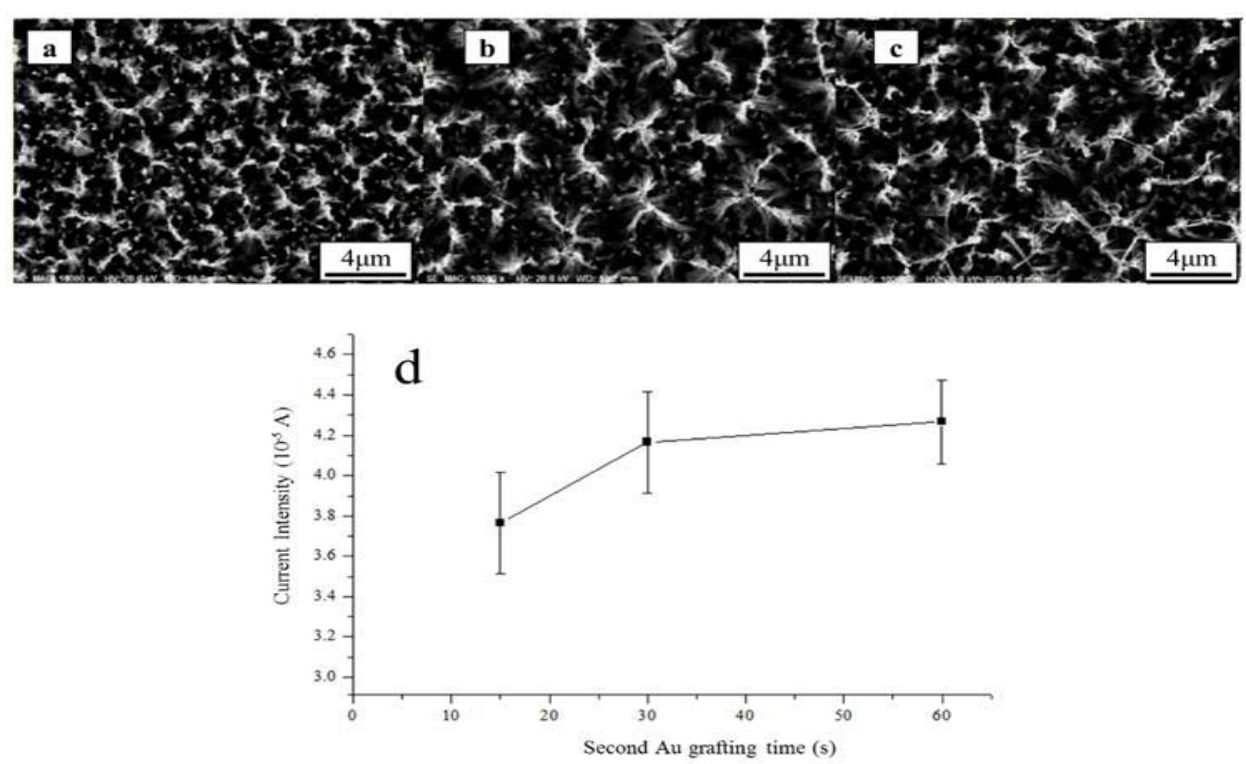

Figure 4: Top SEM images showing nanostructured silicon surface morphologies with second Au decoration/deposition time of (a) 15 (b) 30 and (c) 60 seconds. (d) Shows electrochemical current intensity with second Au decoration/deposition time. It is noted that the first Au electroless deposition time was 30 , and metalassisted etching time was 900 seconds. The glucose sample concentration is $10^{-5} \mathrm{M}$.
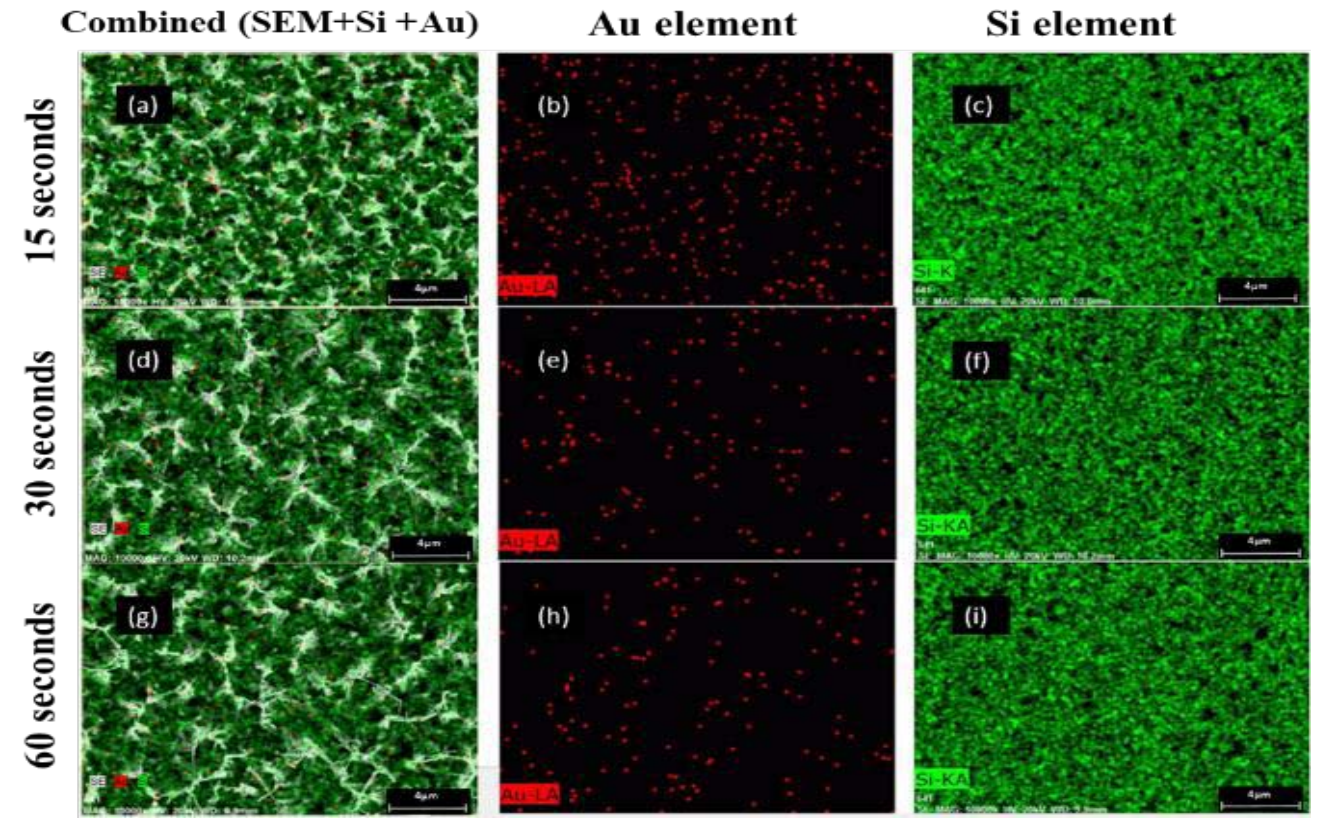

Figure 5: SEM/EDS elemental mapping of nanostructure silicon top surface with 15 seconds (a c), 30 seconds (d f), and 60 seconds ( $\mathrm{g}$ i) Au decoration/ deposition time.

time of 15 seconds, more gold nanoparticles are decorated on the nanostructure surface with bigger pore size surface morphology. This promotes better electrochemical sensing performance due to more reaction sites (electrode area) for higher current intensity.

Figure 3 displayed cross-sectional SEM images of nanostructured silicon surface with different etching times. As the etching times increase, the etching depth and the pore width also increase with the etching time. Although the silicon morphology becomes random at the nanostructure bottom, the silicon nanostructures (red-dash box in Figure 2) were uniformly distributed on the top surface. The gold nanoparticles are decorated on the top size, where electrochemical sensing and MS analysis take place. From Figure 2, it can be observed that gold nanoparticles (red-color dots) are uniformly distributed on the silicon nanostructures (green-color clusters).

\section{Effects of Au decoration/deposition time to electrochemical sensing}

The Au nanoparticles on the $\mathrm{nSi}$ surface acts as reaction sites for electrochemical sensing. Thus, the second Au decoration/deposition 


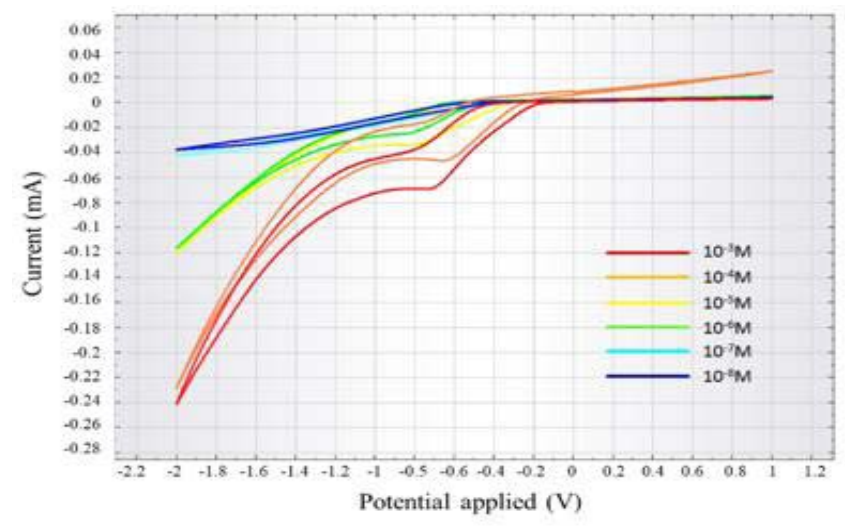

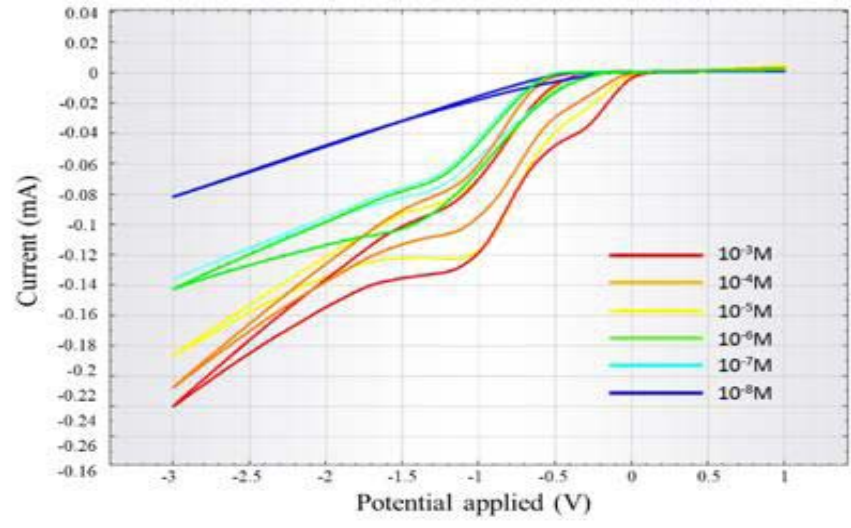

b

Figure 6: $\mathrm{CV}$ curves for (a) 15 and (b) 30 seconds Au decoration condition with glucose concentration from $10^{-3}$ to $10^{-8} \mathrm{M}$. The nSi surface was fabricated from optimized 15 seconds first electroless etching and 900 seconds metal-assisted etching time.

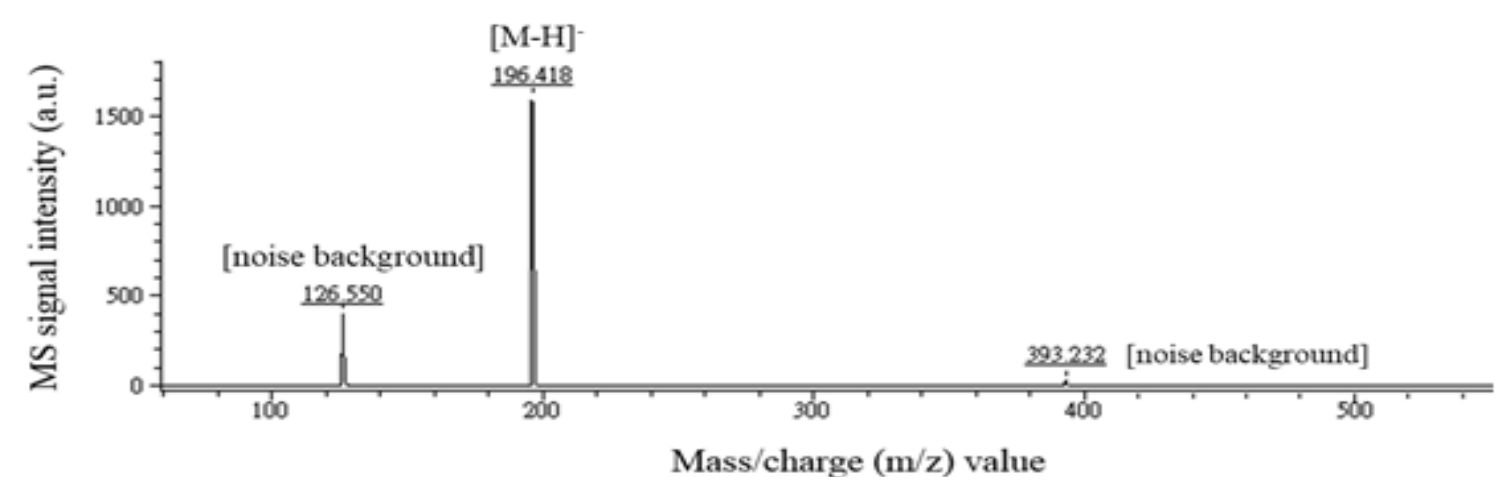

Figure 7: nSi-MS analysis of glucose sample using the nanostructure silicon surface. Glucose sample concentration: $10^{-8} \mathrm{M}[14]$.

time may potentially affect the electrochemical sensing. Figure $4 \mathrm{a}-$ $4 \mathrm{c}$ shows the $\mathrm{nSi}$ surface morphologies with second Au decoration/ deposition time from 15 to 60 seconds. During the second electroless Au grating steps, the surface pore size also increased with decoration/ deposition time from $\sim 2.0 \mu \mathrm{m}$ (Figure $4 \mathrm{a}$ ) to $\sim 4.0 \mu \mathrm{m}$ (Figure $4 \mathrm{c}$ ). The current density also increased from $\sim 3.8 \times 10^{-5} \mathrm{~A}$ to $\sim 4.2 \times 10^{-5} \mathrm{~A}$. This phenomenon is similar to the observation of the etching time effects as previously discussed. Due to larger pore sizes and longer $\mathrm{Au}$ decoration/deposition time, more Au nanoparticles were decorated on the nSi surfaces, which increases the working electrode area for higher electrochemical sensitivity.

Figure 5 shows the SEM/EDS elemental analysis of the nanostructured silicon surface with 15,30 , and 60 seconds $\mathrm{Au}$ decoration/deposition time. Au nanoparticles were uniformly decorated on the nSi surface as expected. And more Au nanoparticles are decorated on the $\mathrm{nSi}$ surface with longer decoration/deposition time. The Au element weight percentage increased from $1.88 \%$ (15 seconds), $2.33 \%$ (30 seconds) to $3.18 \%$ (60 seconds) as displayed in Figure 5 .

Detection sensitivity of electrochemical sensing and LDIMS on nanostructured silicon platform

After evaluating the key process parameter effects to the nSi electrochemical sensing performance, the detection sensitivity was finally evaluated. Figure 6 shows CV curves for 15 seconds (Figure 6a) and 30 seconds (Figure 6b) Au decoration/deposition nSi surface with glucose sample concentration ranging from $10^{-3} \mathrm{M}$ to $10^{-8} \mathrm{M}$. Results indicated that high detection sensitivity of $10^{-7} \mathrm{M}$ and 10 ${ }^{6} \mathrm{M}$ were achieved for 15 and 30 seconds conditions. Comparing to current high sensitive electrochemical glucose biosensors with a limit of detection from $1 \sim 10 \times 10^{-6} \mathrm{M}$ range [34,35], the nSi-based electrochemical sensor exhibits good sensing performance.

As demonstrated in previous investigation, the nSi platform is also capable for the used as LDI-MS target substrate. Therefore, both electrochemical sensing and LDI-MS detection can be performed on the same analytical platform. In our previous investigation, we proved the nSi surface detecting glucose sample at high sensitivity [14]. Based on the key nSi process parameters as previously investigated, the $\mathrm{nSi}$ surface fabricated from 30 seconds first electroless deposition, 900 seconds metal-assisted etching, and 15 seconds second Au deposition. The mass spectrum displayed in Figure 7, showing a highly discernible peak at $196 \mathrm{~m} / \mathrm{z}$ indicates the gluconic acid molecules with nSi LDIMS detection sensitivity of 10-8 M on the nSi surface.

\section{Conclusion}

Electrochemical biosensor provides simple, rapid, and lowcost advantages, which is ideal for rapid and high throughput onsite sample screening purposes. In this study, we demonstrate the 
feasibility of using nanostructure silicon surface for electrochemical sensing on the nSi-MS surface based on the same fabrication process. Effects of critical fabrication parameters on the electrochemical sensing were investigated. This integrated $\mathrm{nSi}$ surface is fabricated from the one first electroless Au deposition step followed by metalassisted etching and second electroless Au decoration/deposition. We used standard glucose samples as standard proof-of-concept. Results find that longer metal-assisted etching time and Au decoration/ deposition time enhanced the electrochemical sensing sensitivity. And detection sensitivity of $10^{-6} \mathrm{M}$ for electrochemical sensing on the nSi-MS platform.

\section{Acknowledgments}

Authors would like thank to Ministry of Science and Technology, Taiwan (grant \# MOST 106-2221-E-008-057 and MOST-2221-E008-028-MY2) financially support this project.

\section{References}

1. Glish GL \& Vachet RW. The basics of mass spectrometry in the twenty-first century. Nature Reviews Drug Discovery. 2003; 2: 140.

2. Aksenov AA, Da Silva R, Knight R, Lopes NP \& Dorrestein PC. Global chemical analysis of biology by mass spectrometry. Nature Reviews Chemistry. 2017; 1: 0054

3. Vidova V \& Spacil Z. A review on mass spectrometry-based quantitative proteomics: Targeted and data independent acquisition. Analytica Chimica Acta. 2017; 964: 7-23.

4. Gluckmann $M$ \& Karas $M$. The initial ion velocity and its dependence on matrix, analyte and preparation method in ultraviolet matrix-assisted laser desorption ionization. Journal of Mass Spectrometry. 1999; 34: 467-477.

5. Hosseini S \& Martinez-Chapa SO. Principles and Mechanism of MALDIToF-MS Analysis. Fundamentals of MALDI-ToF-MS Analysis: Applications in Bio-diagnosis, Tissue Engineering and Drug Delivery. Singapore: Springer Singapore. 2017

6. Devoe DL \& Lee CS. Microfluidic technologies for MALDI-MS in proteomics. Electrophoresis. 2006; 27: 3559-3568.

7. Tsao C-W, Chen K-S \& Hwang F-N. Numerical simulation of dropletbased microfluidic chip interfacing with laser desorption/ionisation mass spectrometry target substrate. Micro \&amp; Nano Letters. 2015; 10.

8. Wei J, Buriak JM \& Siuzdak G. Desorption-ionization mass spectrometry on porous silicon. Nature. 1999; 399: 243-246.

9. Trauger SA, Go EP, Shen Z, Apon JV, Compton BJ, Bouvier ESP, et al. High Sensitivity and Analyte Capture with Desorption/lonization Mass Spectrometry on Silylated Porous Silicon. Analytical Chemistry. 2004; 76 : 4484-4489.

10. Go EP, Apon JV, Luo G, Saghatelian A, Daniels RH, Sahi V, et al. Desorption/ Ionization on Silicon Nanowires. Analytical Chemistry. 2005; 77: 1641-1646.

11. Kurylo I, Dupre M, Cantel S, Enjalbal C, Drobecq H, Szunerits S, et al. Characterization of peptide attachment on silicon nanowires by X-ray photoelectron spectroscopy and mass spectrometry. Analyst. 2017a; 142: 969-978

12. Chen WY, Huang JT, Cheng YC, Chien CC \& Tsao CW. Fabrication of nanostructured silicon by metal-assisted etching and its effects on matrixfree laser desorption/ionization mass spectrometry. Anal Chim Acta. 2011; 687: 97-104

13. Tsao C-W, Kumar P, Liu J \& Devoe DL. Dynamic Electrowetting on Nanofilament Silicon for Matrix-Free Laser Desorption/lonization Mass Spectrometry. Analytical Chemistry. 2008; 80: 2973-2981.

14. Tsao C-W \& Yang Z-J. High Sensitivity and High Detection Specificity of GoldNanoparticle-Grafted Nanostructured Silicon Mass Spectrometry for Glucose Analysis. ACS Applied Materials \& Interfaces. 2015; 7: 22630-22637.
15. Seino $T$, Sato $H$, Yamamoto $A$, Nemoto $A$, Torimura $M$ \& Tao $H$. MatrixFree Laser Desorption/Ionization-Mass Spectrometry Using Self-Assembled Germanium Nanodots. Analytical Chemistry. 2007; 79: 4827-4832.

16. Chen S, Zheng H, Wang J, Hou J, He Q, Liu H, et al. Carbon Nanodots As a Matrix for the Analysis of Low-Molecular-Weight Molecules in Both Positiveand Negative-Ion Matrix-Assisted Laser Desorption/Ionization Time-of-Flight Mass Spectrometry and Quantification of Glucose and Uric Acid in Real Samples. Analytical Chemistry. 2013; 85: 6646-6652.

17. Wang Y, Zeng Z, Li J, Chi L, Guo X \& Lu N. Biomimetic Antireflective Silicon Nanocones Array for Small Molecules Analysis. Journal of the American Society for Mass Spectrometry. 2013; 24: 66-73.

18. Gulbakan B, Park D, Kang M, Kececi K, Martin CR, Powell DH, et al. Laser Desorption Ionization Mass Spectrometry on Silicon Nanowell Arrays. Analytical Chemistry. 2010; 82: 7566-7575

19. Morris NJ, Anderson H, Thibeault B, Vertes A, Powell MJ \& Razunguzwa TT. Laser desorption ionization (LDI) silicon nanopost array chips fabricated using deep UV projection lithography and deep reactive ion etching. RSC Advances. 2015; 5: 72051-72057.

20. Shi CY \& Deng CH. Recent advances in inorganic materials for LDI-MS analysis of small molecules. Analyst. 2016; 141: 2816-2826.

21. Chu H-W, Unnikrishnan B, Anand A, Mao J-Y \& Huang C-C. Nanoparticlebased laser desorption/ionization mass spectrometric analysis of drugs and metabolites. Journal of Food and Drug Analysis. 2018; 26: 1215-1228.

22. Lewis WG, Shen Z, Finn MG \& Siuzdak G. Desorption/ionization on silicon (DIOS) mass spectrometry: background and applications. International Journal of Mass Spectrometry. 2003; 226: 107-116.

23. Thomas JJ, Shen Z, Crowell JE, Finn MG \& Siuzdak G. Desorption/ ionization on silicon (DIOS): A diverse mass spectrometry platform for protein characterization. Proceedings of the National Academy of Sciences. 2001; 98: 4932-4937.

24. Tsao CW, Chen WY, Lin CH \& Cheng YC. Nanostructured Silicon Substrate for Desorption/Ionization on Silicon Mass Spectrometry Coupled with Titanium Oxide and Zinc Oxide Coated Magnetic Nanoparticles for Phosphopeptide Analysis. Journal of Nanoscience and Nanotechnology. 2017; 17: 2054-2060.

25. Kurylo I, Hamdi A, Addad A, Boukherroub R \& Coffinier Y. Comparison of Ti-Based Coatings on Silicon Nanowires for Phosphopeptide Enrichment and Their Laser Assisted Desorption/Ionization Mass Spectrometry Detection. Nanomaterials. 2017b; 7.

26. Tata A, Fernandes AM, AP, Santos VG, Alberici RM, Araldi D, et al. Nanoassisted Laser Desorption-Ionization-MS Imaging of Tumors. Analytical Chemistry. 2012; 84: 6341-6345.

27. Cheng YC, Chen KH, Wang JS, Hsu WL, Chien CC, Chen WY, et al. Rapid analysis of abused drugs using nanostructured silicon surface assisted laser desorption/ionization mass spectrometry. Analyst. 2012; 137: 654-661.

28. Kim SH, Kim J, Jo S-H, Kim J-H, Kim KJ \& Yoon S. Bacterial analysis by laser desorption ionization mass spectrometry on amorphous silicon. Biointerphases. 2016; 11: 041008.

29. Picca RA, Calvano CD, Lo Faro MJ, Fazio B, Trusso S, Ossi PM, et al. Functionalization of silicon nanowire arrays by silver nanoparticles for the laser desorption ionization mass spectrometry analysis of vegetable oils. Journal of Mass Spectrometry. 2016; 51: 849-856.

30. Abdelmaksoud HH, Guinan TM \& Voelcker NH. Fabrication of Nanostructured Mesoporous Germanium for Application in Laser Desorption Ionization Mass Spectrometry. ACS Applied Materials \& Interfaces. 2017; 9: 5092-5099.

31. Grieshaber D, Mackenzie R, VRS J \& Reimhult E. Electrochemical Biosensors - Sensor Principles and Architectures. Sensors (Basel, Switzerland). 2008; 8: 1400-1458.

32. Huang Z, Geyer N, Werner P, De Boor J \& G Sele U. Metal-Assisted Chemical Etching of Silicon: A Review. Advanced Materials. 2011; 23: 285-308.

33. Pasta M, La Mantia F \& Cui Y. Mechanism of glucose electrochemical oxidation on gold surface. Electrochimica Acta. 2010; 55: 5561-5568. 
34. Thanh TD, Balamurugan J, Hwang JY, Kim NH \& Lee JH. In situ synthesis of graphene-encapsulated gold nanoparticle hybrid electrodes for nonenzymatic glucose sensing. Carbon. 2016; 98: 90-98.

35. S Nchez-Obrero G, Cano M, Ávila JL, May NM, Mena ML, Pingarr NJM, et al. A gold nanoparticle-modified PVC/TTF-TCNQ composite amperometric biosensor for glucose determination. Journal of Electroanalytical Chemistry. 2009; 634: 59-63. 